\title{
Evidence of Omega Cen tidal debris in the Kapteyn moving group
}

\author{
Elizabeth Wylie-de Boer ${ }^{1}$, Kenneth Freeman ${ }^{1}$ and Mary Williams ${ }^{2}$ \\ ${ }^{1}$ Research School of Astronomy and Astrophysics, Australian National University \\ Cotter Road, Weston Creek, ACT, Australia 2611 \\ email: ewylie@mso.anu.edu.au, kcf@mso.anu.edu.au \\ ${ }^{2}$ Astrophysikalisches Institut Potsdam, \\ An der Sternwarte 16, D-14482 Potsdam, Germany \\ email: mary@aip.de
}

\begin{abstract}
This paper presents a detailed kinematic and chemical analysis of 16 members of the Kapteyn moving group. The group does not appear to be chemically homogenous. However, the kinematics and the chemical abundance patterns seen in 14 of the stars in this group are similar to those observed in the well-studied cluster, $\omega$ Centauri. Some members of this moving group may be remnants of the tidal debris of $\omega$ Cen, left in the Galactic disk during the merger event which deposited $\omega$ Cen into the Milky Way. A more detailed version of this work can be found in Wylie de-Boer et al. 2009.
\end{abstract}

Keywords. stars: abundances, stars: kinematics, globular clusters: individual( $\omega$ Centauri), Galaxy: structure

\section{Introduction}

The most massive Galactic globular cluster, $\omega$ Cen, has several unique physical properties which suggest that there are very significant differences in star formation histories, enrichment processes and structure formation between $\omega$ Cen and other normal globular clusters Bekki \& Freeman (2003). A commonly accepted scenario of formation of $\omega$ Cen is that it is the surviving nucleus of an ancient dwarf galaxy, the outer envelope of which was entirely removed by tidal stripping as it was accreted by the Galaxy (Bekki \& Freeman (2003) and references therein). Dinescu (2002) also provided a theoretical prediction of where the $\omega$ Cen group would appear kinematically using the metal-poor star sample of Beers et al. (2000). This region covers a large interval of energy over an angular momentum range from about $\mathrm{L}_{z}=-200$ to $-600 \mathrm{kpc} \mathrm{km} \mathrm{s}^{-1}$.

We have recently investigated the chemical properties of stars in the Kapteyn moving group, first introduced by Eggen (1962). The stars of the Kapteyn group, as most recently tabulated by Eggen (1996), are mostly metal-poor and in retrograde galactic orbits, so they were identified as a halo moving group. It turned out that some of the group stars show chemical peculiarities similar to those seen in $\omega$ Cen, and we will argue that the Kapteyn group may be part of the $\omega$ Cen debris.

\section{Kinematics Results}

The Lindblad diagram is shown in Figure 1 for the Kapteyn group stars and the Gratton peak sample from Gratton et al. (2003). The large open circles represent the Gratton peak stars. The large solid circles are for the retrograde Kapteyn group stars and the elongated cloud of smaller coloured points around each solid circle show the outcome 
of the Monte Carlo simulations on uncertainties, and outline the $1-\sigma$ confidence level for each Kapteyn group star. As expected, the errors in $\mathrm{L}_{z}$ and $\mathrm{E}$ are highly correlated. The open star is for $\omega$ Cen. The region in which Dinescu (2002) suggested that candidate stars from Omega Cen's host galaxy could lie is outlined by the dashed line. This range depends on the adopted velocity of the Local Standard of Rest (taken here to be $220 \mathrm{~km} \mathrm{~s}^{-1}$ ). The dotted lines indicate the Dinescu range using recent extreme values of the LSR velocity.

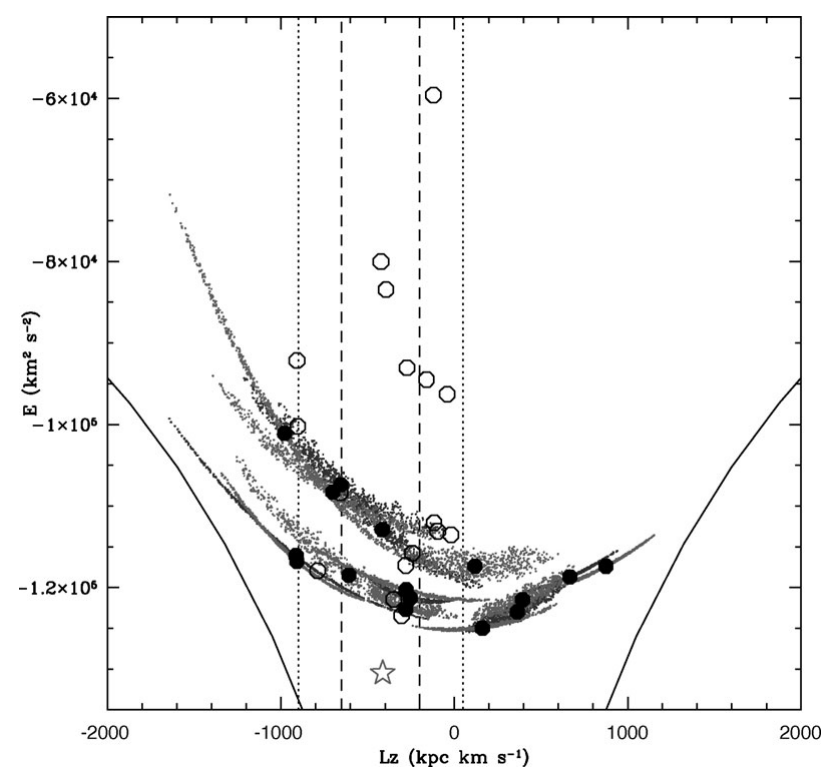

Figure 1. Lindblad diagram for Kapteyn group and Gratton peak. The black filled circles are the 16 Kapteyn values and the open star indicates the position of $\omega$ Centauri. The black open circles are stars from the Gratton $\omega$ Cen peak. The smaller dots represent the error distribution in $\mathrm{E}, \mathrm{L}_{z}$ for the Kapteyn group stars, as discussed in the text. The different colors used are intended only to provide clarity between the distributions for different stars.

The Kapteyn group stars in Figure 1 appear to lie mainly in two bands. The appearance of these bands is accentuated by the correlated errors on $\mathrm{E}$ and $\mathrm{L}_{z}$. Figure 1 shows that nine of the Kapteyn stars lie within the box covering the Dinescu et al. region of $\omega$ Cen candidates. Within the 1- $\sigma$ uncertainties, this number increases to 12 members of the Kapteyn group. There are four prograde stars in the Kaptyen sample. Two of these stars lie such that the ends of their 1- $\sigma$ distribution fall near the extreme of the $\omega$ Cen region, leaving only two stars (HD13979 and CD -30 1121) which are clearly separate from the $\omega$ Cen candidate region.

In the following analysis, the 16 Kapteyn stars are partitioned into two groups. From Figure 1, fourteen stars are taken as kinematically associated with $\omega$ Cen, while two of the stars are likely to have no kinematic connection to $\omega$ Cen debris.

\section{Abundance Results}

First we compare the abundances in $\omega$ Cen and in the field halo stars. Then we compare the Kapteyn group abundances with those in the cluster and the field halo, in order to 
ascertain whether the Kapteyn group stars show the abundance patterns of either of these two populations. The main point of interest here is that the Kapteyn stars which appear to be kinematically related to $\omega$ Cen share distinct abundance similarities with those stars from previous $\omega$ Cen studies. The abundances for the potential $\omega$ Cen candidates among the Kapteyn stars could be drawn from the same abundance distribution as the $\omega$ Cen stars, although we note that there is much more scatter in the abundance distributions for most elements in the $\omega$ Cen stars. Whatever the reason for this large scatter in the cluster star abundances, it seems clear that the Kapteyn candidates show abundance anomalies relative to the field halo which are similar to those shown by the stars of $\omega$ Cen. The similarities between the Kapteyn candidates and the $\omega$ Cen stars, relative to the field halo, are clearest in $\mathrm{Na}, \mathrm{Mg}$ and the s-elements.

Although the copper abundance was derived for only five of the stars in our sample, the results are worth mentioning. $\omega$ Cen is known for its unique copper signature. At a constant value of $[\mathrm{Cu} / \mathrm{Fe}] \sim-0.6$, it is distinct from the halo stars in the same metallicity range, which show an increase with metallicity up to $[\mathrm{Cu} / \mathrm{Fe}]=-0.3$ as discussed by Smith et al. (2000). Of the four stars for which copper was measured in our sample, all show deficiencies, with $[\mathrm{Cu} / \mathrm{Fe}]$ values between -0.47 and -0.60 . This adds weight to the case that this group of stars come from the same population as $\omega$ Cen.

Statistical tests were undertaken on the abundance distributions to determine the likelihood that the three groups of stars (Kapteyn group, $\omega$ Cen and the halo) come from the same population. The $\omega$ Cen group consisted of stars from the Norris \& Da Costa (1995) and Smith et al. (2000) studies and includes the stars from Gratton et al. (2003) only for elements in common with this study, $\mathrm{Na}, \mathrm{Mg}$ and $\mathrm{Ca}$. The halo group is taken from the Venn et al. (2004) compilation and is defined as being any halo population star that falls within the plausible range of $[\mathrm{Fe} / \mathrm{H}]$ values for $\omega$ Cen of $[\mathrm{Fe} / \mathrm{H}]=-0.5$ to -2.5 . Figure 2 shows the mean and standard deviation of the mean, $\sigma_{\text {mean }}$, graphically for the five elements studied, with the Kapteyn group shown as filled red squares, the $\omega$ Cen stars as filled magenta triangles and the field halo stars as open green circles. It is evident from this figure that in all elements, except $\mathrm{Ca}$ and to a lesser extent $\mathrm{Mg}$, the field halo stars are chemically distinct from $\omega$ Cen. It is also clear that, except for Ca, the Kapteyn group and $\omega$ Cen populations overlap in $[\mathrm{X} / \mathrm{Fe}]$.

\section{Discussion and Conclusion}

The kinematic and abundance analysis in this study suggests that at least our 14 members of the Kapteyn group and potentially many more stars in retrograde orbits which were not observed in this study, could be remnants of tidal debris stripped from the parent galaxy of $\omega$ Cen, or even from the cluster itself, during its merger with the Galaxy.

Our study provides the first detailed chemical evidence of field stars that appear to be both kinematically and chemically related to $\omega$ Cen. It may lend weight to the view that $\omega$ Cen is the remnant nucleus of a disrupted dwarf galaxy which was accreted by the Milky Way, by providing chemical evidence of tidal debris among the Galactic field stars.

The three-banded structure seen in the Lindblad diagrams of Figures 3 and described in Section 2.1 may be indicative of stars shed with different energies from different wraps of the decaying orbit of the parent galaxy around the Milky Way. The reader is referred to Figure 6 in Meza et al. (2005) which shows several distinct $\mathrm{E}-\mathrm{L}_{z}$ curves from their numerical simulations of merger debris. The presence of this banded structure, along with the present Galactic radius of $\omega$ Cen, suggest the original parent galaxy was relatively 

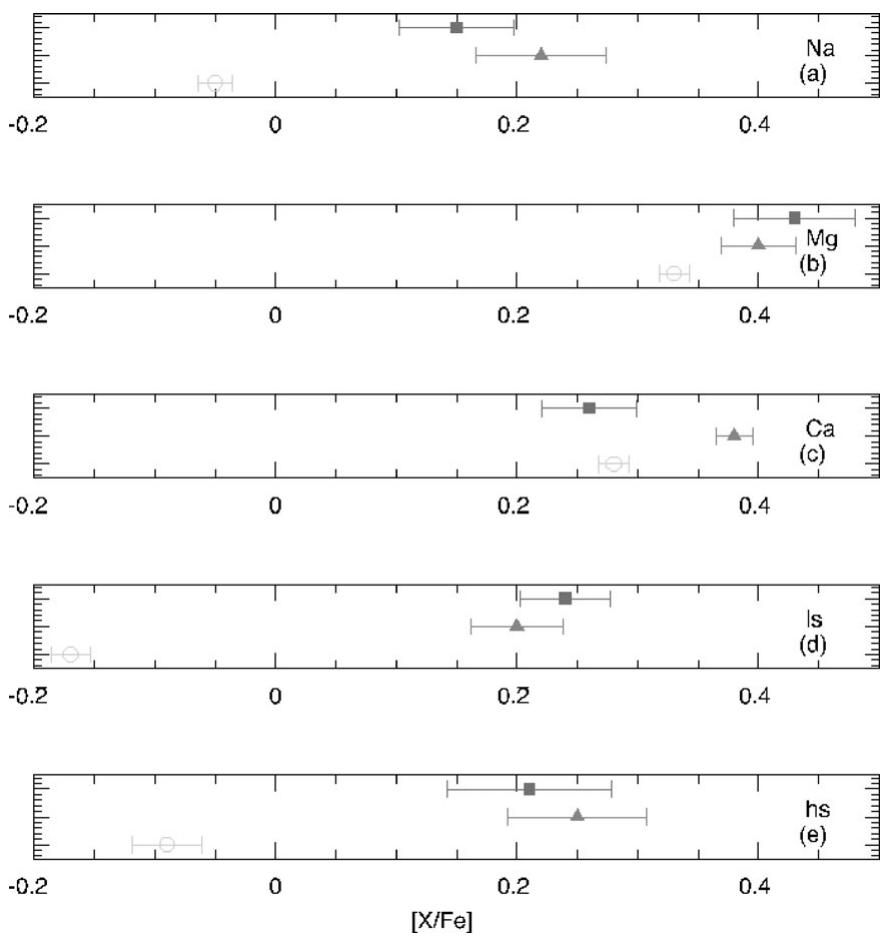

Figure 2. The mean abundance and standard deviation of the mean abundance of the Kapteyn group stars (filled squares), $\omega$ Cen stars (filled triangles)and halo stars (open circles) for the five different elements studied: a) $[\mathrm{Na} / \mathrm{Fe}]$, b) $[\mathrm{Mg} / \mathrm{Fe}]$, c) $[\mathrm{Ca} / \mathrm{Fe}]$, d) $[\mathrm{ls} / \mathrm{Fe}]$ and e) $[\mathrm{hs} / \mathrm{Fe}]$. It is easy to see the mean abundance and distributions of each population and compare to the others. The $\mathrm{y}$ axis simply spreads the three different groups out for clarity and ease of comparison.

massive in order for dynamical friction to have the required effect, and was also relatively dense in order to survive the Galactic tidal stresses in to the current orbital radius of $\omega$ Cen.

\section{References}

Beers, T. C., Chiba, M., Yoshii, Y., Platais, I., Hanson, R. B., Fuchs, B., \& Rossi, S. 2000, AJ, 119,2866

Bekki, K. \& Freeman, K. C. 2003, MNRAS, 346, L11

Dinescu, D. I. 2002, in Astronomical Society of the Pacific Conference Series, Vol. 265, Omega Centauri, A Unique Window into Astrophysics, ed. F. van Leeuwen, J. D. Hughes, \& G. Piotto, 365-+

Eggen, O. J. 1996, AJ, 112, 1595

Eggen, O. J., Lynden-Bell, D., \& Sandage, A. R. 1962, ApJ, 136, 748

Gratton, R. G., Carretta, E., Desidera, S., Lucatello, S., Mazzei, P., \& Barbieri, M. 2003, A\&\&A, 406,131

Meza, A., Navarro, J. F., Abadi, M. G., \& Steinmetz, M. 2005, MNRAS, 359, 93

Norris, J. E. \& Da Costa, G. S. 1995, ApJ, 447, 680

Smith, V. V., Suntzeff, N. B., Cunha, K., Gallino, R., Busso, M., Lambert, D. L., \& Straniero, O. $2000, A J, 119,1239$

Venn, K. A., Irwin, M., Shetrone, M. D., Tout, C. A., Hill, V., \& Tolstoy, E. 2004, AJ, 128, 1177

Wylie-de Boer, E. C., Freeman, K. C., \& Williams, M. 2009, AJ, in press 